University of Nebraska - Lincoln

DigitalCommons@University of Nebraska - Lincoln

8-1-2001

\title{
Yang-Lee edge singularities determined from experimental high- field magnetization data
}

Christian Binek

University of Nebraska-Lincoln, cbinek@unl.edu

Wolfgang Kleemann

Laboratorium für Angewandte Physik, Gerhard-Mercator-Universität Duisburg, D-47048 Duisburg,

Germany, wolfgang.kleemann@uni-due.de

H. Aruga Katori

RIKEN (The Institute of Physical and Chemical Research), Wako, Saitama 351-0198, Japan

Follow this and additional works at: https://digitalcommons.unl.edu/physicsbinek

Part of the Physics Commons

Binek, Christian; Kleemann, Wolfgang; and Aruga Katori, H., "Yang-Lee edge singularities determined from experimental high-field magnetization data" (2001). Christian Binek Publications. 34.

https://digitalcommons.unl.edu/physicsbinek/34

This Article is brought to you for free and open access by the Research Papers in Physics and Astronomy at DigitalCommons@University of Nebraska - Lincoln. It has been accepted for inclusion in Christian Binek Publications by an authorized administrator of DigitalCommons@University of Nebraska - Lincoln. 
Published in Journal of Physics: Condensed Matter 13 (2001), pp. L811-L817. Copyright (C) 2001 Institute of Physics Publishing Ltd. Used by permission. Online at stacks.iop.org/JPhysCM/13/L811

Submitted August 2, 2001; published August 16, 2001

LETTER TO THE EDITOR

\title{
Yang-Lee edge singularities determined from experimental high-field magnetization data
}

\author{
C. Binek ${ }^{1}$, W. Kleemann ${ }^{1}$, and H. Aruga Katori ${ }^{2}$ \\ ${ }^{1}$ Laboratorium für Angewandte Physik, Gerhard-Mercator-Universität Duisburg, \\ D-47048 Duisburg, Germany \\ ${ }^{2}$ RIKEN (The Institute of Physical and Chemical Research), Wako, Saitama 351-0198, Japan
}

\begin{abstract}
The isothermal magnetization $m(H)$ of the metamagnet $\mathrm{FeCl}_{2}$ is measured in axial magnetic fields $0 \leq \mu_{0} H_{a} \leq 12 \mathrm{~T}$ at temperatures $34 \leq T \leq 53 \mathrm{~K}$ above the Néel temperature, where the system is essentially a two-dimensional Ising ferromagnet. The analysis of the data indicates experimental accessibility of the critical exponent $\mu$ of the Yang-Lee edge singularities. They manifest themselves in divergences of the density functions $g(\theta)$, which quantify the distribution of the zeros of the partition function on the Lee-Yang unit circle in the complex plane. In accordance with the hypothesis of universality, a critical exponent close to the theoretical prediction for the two-dimensional Ising ferromagnet, $\mu=-1 / 6$, is found.
\end{abstract}

The polynomial representation of the partition function $Z$ of an Ising system enables the complete determination of its thermodynamic behavior from the knowledge of the complex zeros of $Z(z)=0$. Here the variable $z=\exp \left(-2 g S \mu_{B} \mu_{0} H / k_{B} T\right)$, whose powers build up the polynomial $Z$, contains the magnetic moment $g S \mu_{B}$ of the Ising spin $S= \pm 1$ with Landé factor $g$, the complex magnetic field $H$ and the temperature $T$. In their profound analysis of the Ising model, Lee and Yang [1] discovered a fundamental rigorous result which is known as the "circle theorem" and later turned out to be applicable to much wider classes of model systems [2,3]. It predicts that the zeros of the partition function of an Ising ferromagnet (or a lattice gas) are distributed on a unit circle, $z=$ $\exp (\mathrm{i} \theta)$, in the complex $z$-plane. Within this description the density function of the zeros, $\mathrm{g}(\theta)$, is a crucial quantity. Above the critical temperature $T_{c}$ of the Ising ferromagnet, a gap angle $\theta_{g}>0$ separates all complex zeros from the real axis of the $z$-plane; hence $g(\theta)=0$ for $|\theta|<\theta_{g}$. The gap angle $\theta_{g}$ depends on temperature and becomes zero at $T=T_{c}$, where complex zeros touch the real axis.

The zero density $g(\theta=0)$ which evolves at $T<T_{c}$ determines the spontaneous normalized magnetization $I=\mathrm{m} / \mathrm{m}_{s}$ of the Ising ferromagnet according to $I=2 \pi g(0)$. Here $m$ and $m_{s}$ denote the absolute and saturation magnetization, respectively. At the critical temperature the dependence of the magnetization on small fields is given by $m \propto H^{1 / \delta}$ [4]. In the case of $\delta>1$, this scaling behavior generates a singularity of the magnetic susceptibility $\chi=\partial m / \partial H$ at $H=0$. The non-analytic behav- 
ior of the magnetization at $T=T_{c}$ originates from the accumulation of complex zeros on the real axis at $z(H=0)=1$ [5]. In analogy with the non-analytic behavior of $m$ at $T=T_{c}$ for $H=0$, nonanalyticity also arises at $T>T_{c}$ for the pure imaginary field $H_{0}(T)=-\mathrm{i} \theta \theta_{g} T /\left(2 g S \mu_{B} \mu_{0}\right)$, which defines the onset of the density of zeros $g(\theta)>0$ at $z\left(H=H_{0}\right)=\exp \left(\mathrm{i} \theta_{\mathrm{g}}\right)$ [6]. On approaching $\theta_{\mathrm{g}}$ from above, the density function exhibits a power-law behavior $g \propto\left(\theta-\theta_{g}\right)^{\mu}$, which is the manifestation of the Yang-Lee edge singularity. In accordance with this singularity at $\theta=\theta_{g}$, the generalized susceptibility also diverges at $H=H_{0}$ in the case of $\mu<1$. Both $\mu<1$ and the universality of $\mu$ are assumed to hold in general [7]. In the case of a two-dimensional (2D) Ising ferromagnet, a rigorous theory predicts $\mu=-1 / 6$ [8].

Only recently, we showed that the density functions of a 2D Ising ferromagnet on a triangular lattice are experimentally accessible by analysing the isothermal magnetization data, $m$ versus $H$, of $\mathrm{FeCl}_{2}$ [9]. To the best of our knowledge, this is the only experimental work that has been carried out up to now [10]. However, the limitation of the available magnetic field strength of our superconducting quantum interference device (SQUID), $\mu_{0} H=5 \mathrm{~T}$, prevents us from revealing the details of the density function. In particular, the edge singularity was smeared out into a barely pronounced maximum. Thus one of the most important predictions, namely the power-law criticality, $g \propto\left(\theta-\theta_{g}\right)^{\mu}$, with the universal exponent $\mu<1$ [7], remained unconfirmed. It is the aim of this letter to present and analyse new magnetization data, which are obtained from vibrating-sample magnetometer (VSM) measurements in axial magnetic fields up to $\mu_{0} H=12 \mathrm{~T}$. The enlarged field range on the one hand and an appropriate refinement of the ansatz function on the other hand indicate that the determination of the critical exponent $\mu$ of the Lee-Yang edge singularity from experimental data is possible.

It should be noticed that the analysis is applicable only to a limited temperature range, where the $2 \mathrm{D}$ ferromagnetic properties of $\mathrm{FeCl}_{2}$ are prevalent on the one hand and $\theta_{g}$ is sufficiently small in comparison with the high-temperature limit, $\theta_{g}=\pi$, on the other hand. The latter condition ensures that the real experimental magnetization data are not too far away from the singularity in the complex plane.

The experiments are carried out by VSM measurements (Oxford Instruments MagLab ${ }^{\mathrm{VSM}}$ ) on an as-cleaved square $c$-platelet with thickness $t=0.3 \mathrm{~mm}$ and area $A=4 \mathrm{~mm}^{2}$. The sample is mounted in a small gelatin capsule. The capsule is filled with cotton wool in order to prevent any movement of the sample. The magnetic moment of the sample holder turns out to be less than $0.2 \%$ of the magnetic moment of the sample at $T=50 \mathrm{~K}$ and $\mu_{0} H=12 \mathrm{~T}$. Hence, no background correction of the data has to be taken into account within the analysis.

Figure 1 shows the isothermal magnetization for $T=4,34,49,50$ (inset (a)), 51, 52 (inset (b)) and $53 \mathrm{~K}$ in internal axial magnetic fields $0 \leq \mu_{0} H \leq 12 \mathrm{~T}$. At $T=4 \mathrm{~K} \mathrm{FeCl}_{2}$ undergoes a field-driven metamagnetic transition at $\mu_{0} H_{a} \approx 1 \mathrm{~T}$ from its antiferromagnetic ground state into the paramagnetic saturated state [11]. These $m$ versus $H$ data allow us to determine the saturation magnetic moment $m_{s}$ as well as the demagnetizing factor $N=1 /(\mathrm{d} m / \mathrm{d} H)=$ constant. While $m_{s}$ is used for the normalization of the magnetization isotherms at $34 \mathrm{~K} \leq T \leq 53 \mathrm{~K}, N$ allows us to convert the applied field $H_{a}$ into the internal field $H=H_{a}-N \mathrm{~m}$. Note that the number of data points plotted in figure 1 is reduced by a factor of 50 for $T=4 \mathrm{~K}$ and by a factor of 10 for the data sets at $34 \mathrm{~K} \leq T \leq$ $53 \mathrm{~K}$ with respect to the total number of data points measured and analysed. Above the Néel temperature $T_{N}=23.7 \mathrm{~K}_{\text {of }} \mathrm{FeCl}_{2}$, the $3 \mathrm{D}$ antiferromagnetic long-range order is broken and the system behaves essentially like a 2D Ising ferromagnet on a triangular lattice [12,13]. The dominance of the $2 \mathrm{D}$ character is ensured by the high ratio $J /\left|J^{\prime}\right| \approx 22$ of the intraplanar ferromagnetic interaction $J$ and the antiferromagnetic interplanar interaction $J$ '. Hence, far above $T_{N}, 3 \mathrm{D}$ antiferromagnetic $\mathrm{FeCl}_{2}$ is an appropriate model system in order to study $2 \mathrm{D}$ ferromagnetism. 


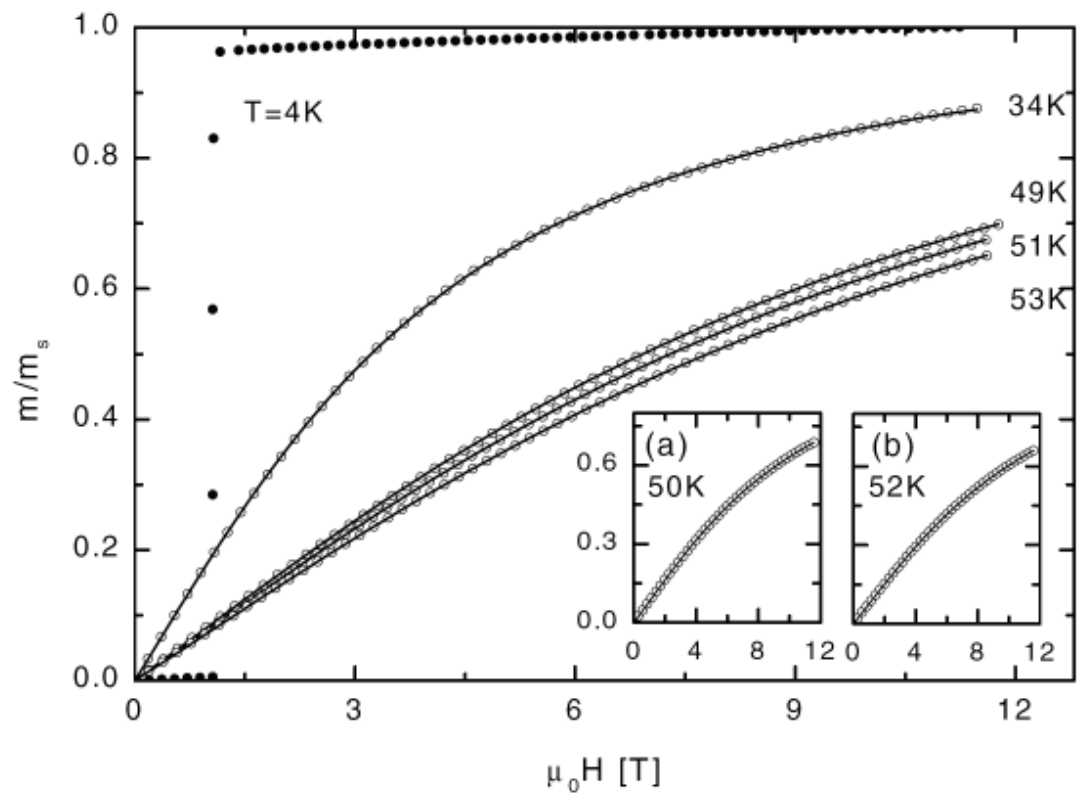

Figure 1. Isothermal magnetization data $m / m_{s}$ versus $H$ for $\mathrm{FeCl}_{2}$ for temperatures $T=4$, 34, 49, 50 (inset (a)), 51, 52 (inset (b)) and $53 \mathrm{~K}$. The densities of data points plotted are reduced by a factor of 50 for $T=4 \mathrm{~K}$ and by a factor of 10 for $34 \mathrm{~K} \leq T \leq 53 \mathrm{~K}$. Best fits of equation ( $\underline{3}$ ) to the data sets for $T \geq 34 \mathrm{~K}$ are shown by solid lines.

As pointed out by Kortman and Griffiths [14], the ansatz function

$$
m \propto \tau\left(\tau^{2}+\tan ^{2}\left(\theta_{g} / 2\right)\right)^{\mu}
$$

with $\tau=(1-z) /(1+z)$ implies a density function with an asymptotic behavior of the type $g \propto(\theta$ $\left.-\theta_{g}\right)^{\mu}$ on approaching $\theta_{g}$ from above. It is, hence, a good candidate for being an appropriate fitting function. Unfortunately, in the case of $\mu>-1 / 2$ an artificial singularity of the density function at $\theta=\pi$ originates from the divergence of the function $\tau(z)$ at $z=\exp (\mathrm{i} \pi)=-1$. Therefore, we modify the function $\tau(z)$ in such a way that the singularity of $g(\theta)$ at $\theta=\pi$ is suppressed. However, the new function $\tilde{\tau}(z)$ still has to conserve both the basic property $\tilde{\tau}(z=1)=0$ and the essential condition $\lim _{\theta \rightarrow \theta_{g}}=-\mathrm{i} \tan \left(\theta_{g} / 2\right)$, which reveals the physical singularity of $g(\theta)$ at $\theta=\theta_{g}$. It can be verified that these conditions are fulfilled most easily by the expression

$$
\tilde{\tau}=(1 / 2)(1-z)\left[1-\frac{\tan \left(\theta_{g} / 2\right)}{\sin \theta_{g}}\left(z-\cos \theta_{g}\right)\right] .
$$

Substitution of equation (2) into the proportionality (1) and normalization of the resulting ansatz function with respect to the saturation magnetization $m_{s}$ yields

$$
I=K \tilde{\tau}\left(\tilde{\tau}+\tan ^{2}\left(\theta_{g} / 2\right)\right)^{\mu} /\left(\tilde{\tau}(0)\left[\tilde{\tau}(0)^{2}+\tan ^{2}\left(\theta_{g} / 2\right)\right]^{\mu}\right) .
$$

Equation (3) is fitted to the $m / m_{s}$ versus $H$ data for $34 \mathrm{~K} \leq T \leq 53 \mathrm{~K}$. In order to take into account small errors which might be involved in the procedure of normalization of the data, a proportionality constant $K \approx 1$ is introduced as a fitting parameter in addition to the physically essential parameters $\mu$ and $\theta_{g}$. In accordance with our previous analysis of SQUID data []ㅡ, the Landé factor, which enters equation ( $\underline{3})$ via the $z$-term of $\tilde{\tau}$, is given by $g=4.1$. 


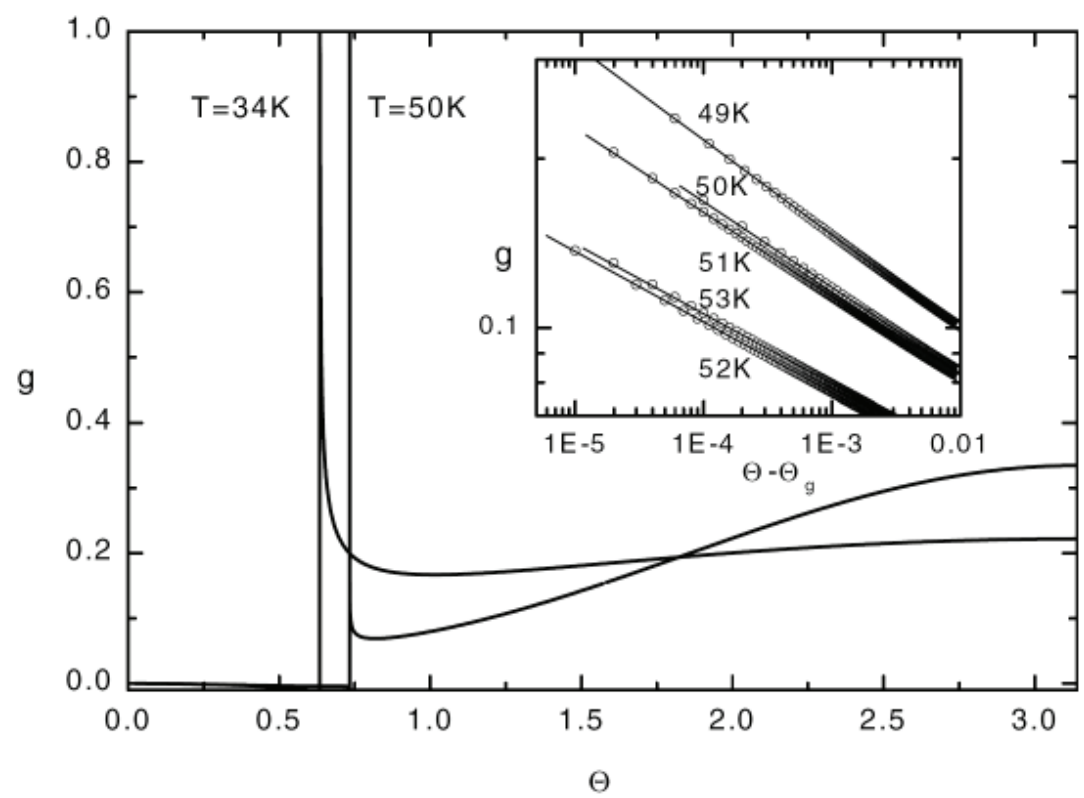

Figure 2. Selected Lee-Yang zero density functions $g(\theta)$ for $T=34$ and $50 \mathrm{~K}$ exhibiting the temperature-dependent density gaps and the Yang-Lee edge singularities. The inset shows the density functions $g$ versus $\theta-\theta_{g}(T)$ for $T=49,50,51,52$ and $53 \mathrm{~K}$ on a $\log -\log$ scale. The slopes of the best-fitted power-law functions (solid lines) yield Yang-Lee edge exponents $\mu=$ $-0.15 \pm 0.02$.

In order to improve the reliability of the parameters obtained from the fitting procedure, the field range investigated has been extended from $5 \mathrm{~T}$ to $12 \mathrm{~T}$ with respect to the SQUID measurements reported in reference [9]. The increased curvature of the isotherms in the high-field regime increases the accuracy and stability of the fitting procedure. Hence, the least-squares fit yields unambiguous sets of parameters within the framework of the ansatz function of equation (3). At temperatures far above $50 \mathrm{~K}$ however, several factors prevent the determination of a meaningful critical exponent. The ferromagnetic exchange energy becomes small in comparison with the thermal energy and the system behaves essentially paramagnetically. In that case all zeros of the ideal paramagnetic Ising system accumulate at $z=-1$ which corresponds to a delta function of the density of zeros, peaking at $\theta=\pi$ [9]. This gap angle quantifies the separation of the singularity from the real positive axis of the complex plane. Hence, one cannot expect to obtain appropriate information about the singularity in the high-temperature limit $\theta_{g} \approx \pi$ from data which are expected to be outside the critical region. Moreover, the Ising-type character of $\mathrm{FeCl}_{2}$ breaks down at high temperatures according to the finite single-ion anisotropy of the $S=1$ effective spin system.

The results of the best fits are shown in figure 1 as solid lines for all of the isotherms at $34 \mathrm{~K} \leq$ $T \leq 53 \mathrm{~K}$. Moreover, figure 2 shows two of the typical density functions for $T=34$ and $50 \mathrm{~K}$. They are calculated by using $[9,14]$

$$
g(\theta)=(1 / 2 \pi) \lim _{r \rightarrow 1^{-}} \operatorname{Re} I(r \exp (\mathrm{i} \theta))
$$

after substitution of the fitting parameters $\mu$ and $\theta_{g}$ into equation (3). As expected, both curves exhibit a gap region of zero density and a pronounced singularity on approaching $\theta_{g}$ from above. Moreover, the gap angle decreases from $\theta_{g}=0.73 \mathrm{rad}$ at $T=50 \mathrm{~K}$ towards $\theta_{g}=0.63 \mathrm{rad}$ at $T=$ 


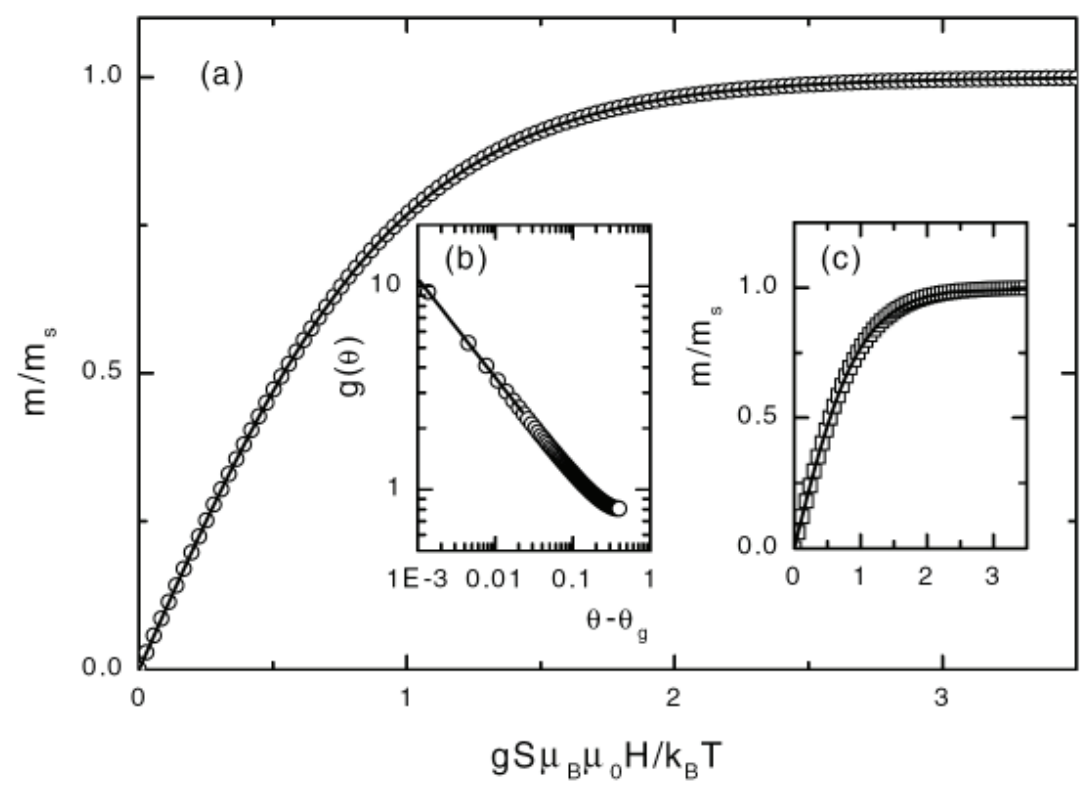

Figure 3. (a) $m / m_{s}$ versus $g S \mu_{B} \mu_{0} H / k_{B} T$ for the linear Ising chain for $k_{B} T / J=100$. The line represents the best fit of equation (1) to the data. Inset (b) shows a log-log plot of the rigorous density function with the linear fit of slope $\mu=-1 / 2$ in the asymptotic regime. Inset (c) shows the magnetization data (squares) with the result of the best fitting of equation (5).

$34 \mathrm{~K}$. This reflects the expected gradual decrease of $\theta_{g}$ with decreasing temperature. The $T$-dependence of $\theta_{g}$ determines the $T$-dependence of $H_{0}(T)$, which defines the critical line of the Yang-Lee edge singularity. Although these critical lines are not universal, a lot of theoretical work has been done in order to determine details of their behavior for various systems $[15,16]$.

Despite the reasonable $T$-dependence of $\theta_{g}$, there is a strong deviation of the Yang-Lee edge exponent $\mu=-0.365$ for $T=34 \mathrm{~K}$ from the value $\mu=-0.156$ which originates from the best fit at $T=$ $50 \mathrm{~K}$. As mentioned above, weak antiferromagnetic superexchange $J \cdot / k_{B}=-0.18 \mathrm{~K}$ between $\mathrm{Fe}^{2+}$ ions on adjacent (111) layers of the rhombohedral $\mathrm{FeCl}_{2}$ crystal gives rise to a crossover into 3D long-range antiferromagnetic order. Although the crossover takes place continuously, a rapid increase of the 3D fluctuations is expected when decreasing the temperature from $T=49 \mathrm{~K}$ to $T=$ $34 \mathrm{~K}$. This precursor phenomenon indicates the subsequent power-law divergence of the antiferromagnetic correlation length near $T_{N}$. This is corroborated by the inset of figure 2 , which shows $g$ versus $\theta-\theta_{g}(T)$ for $T=49,50,51,52$ and $53 \mathrm{~K}$ on a log-log scale. According to the asymptotic power-law behavior of $g(\theta)$ near to $\theta_{g}$, all of the curves show linear behavior with slopes $\mu=-0.15$ \pm 0.02 , in good agreement with the theoretical predictions for $2 \mathrm{D}$ Ising ferromagnets $[7,8]$.

In order to be truly convincing, we have to rule out the possible relevance of simpler theoretical concepts providing satisfactory descriptions of the magnetization isotherms. As an example we choose an exactly solvable model system, namely the linear Ising chain. Here a mean-field approach yields a seemingly good fit to the exact magnetization data which, however, ends up with the wrong gap exponent $\mu=+1 / 2$ according to the ansatz. Figure 3(a) shows the calculated isothermal magnetization (circles) of the linear Ising chain for $k_{B} T / J=100$ [5]. In figure 3(c) these data are fitted to the well-known [17] mean-field equation of an $S=1 / 2$ ferromagnet in arbitrary dimension:

$$
\tanh \left(g S \mu_{B} \mu_{0} H /\left(k_{B} T\right)\right)=\left(m / m_{s}-\tanh \left(m T_{c} /\left(m_{s} T\right)\right)\right) /\left(1-m \tanh \left(m T_{c} /\left(m_{s} T\right)\right) / m_{s}\right) .
$$


Although the fit appears quite satisfactory, two deficiencies become apparent on closer inspection. First, a finite best-fitted value $T_{c} / T=0.02$ is clearly at odds with the non-ordering linear chain. Second, as shown elsewhere [14], the critical behavior attributed to equation (5) yields an exponent $\mu=+1 / 2$ in extreme contrast with the exact one, $\mu=-1 / 2$ [5]. On the other hand, when fitting the data to equation (1) we readily obtain $\mu=-0.50$ and $\theta_{g}=2.74$ (figure 3(a), solid line). Figure 3(b) shows a log-log plot of the density function which is known from reference [5]. The linear fit (line) of slope $\mu=-0.5$ indicates the asymptotic critical behavior of the rigorous expression.

The above example demonstrates that the simplicity of an approach cannot in general be a guideline for the choice of a theoretical description. It is well known from the analysis of critical behavior that in particular a mean-field analysis will rarely provide correct critical exponents although it may sometimes fit the data with sufficient accuracy.

In conclusion, we pointed out that isothermal VSM high-field magnetization data for the prototypical 2D Ising ferromagnet $\mathrm{FeCl}_{2}$ can be used in order to determine the Yang-Lee edge exponent $\mu$. The analysis is based on a modified ansatz function originally suggested by Kortman and Griffiths [14]. It generically contains the possibility of non-analyticity of the magnetization in a purely imaginary field. From best fits of this ansatz function to the magnetization data $m$ versus $H$ we obtain the edge exponent $\mu=-0.15 \pm 0.02$ for all isotherms investigated at $49 \mathrm{~K} \leq T \leq 53 \mathrm{~K}$, in good agreement with the theoretical prediction $[7,8]$. Note, however, that the accuracy of $\mu$ refers to the limited temperature interval where two-dimensionality, Ising anisotropy and FM exchange are optimized. Moreover, the gap angle $\theta_{g}$ exhibits a reasonable temperature dependence. Thus we have pointed out a route which seems to provide experimental access to the theoretical concept of non-analyticity of the magnetization for $T>T_{c}(H=0)$ in a purely imaginary magnetic field which is, of course, experimentally not realizable.

In order to study the criticality of $g(\theta)$ closer to $T_{c}$, future experiments should focus on "real" $2 \mathrm{D}$ Ising ferromagnets as represented by ultrathin layers with uniaxial anisotropy [18]. Possible candidates are, e.g., Co monolayers on $\mathrm{Cu}(111)$ substrates [19] and $\mathrm{Ni}(111)$ layers consisting of less than six monolayers on W(110) [20,21]. Note that the microscopical details of the uniaxial anisotropy are crucial for the selection of an appropriate model system in order to ensure that the anisotropy is maintained at $T>T_{c}$. This cannot be straightforwardly deduced from the phenomenological anisotropy constants which are expected to vanish above $T_{c}$ [22]. Moreover, while investigating $m$ versus $H$ in the temperature range $T>T_{c}$, care has to be taken that the magnetic properties of the filmsubstrate system are not spoilt by thermal interdiffusion [23]. Hence, systems with sufficiently low values of $T_{c}$ have to be chosen.

\section{Acknowledgments}

Ch. Binek would like to thank K. Katsumata for fruitful discussions and kind hospitality enjoyed while carrying out the experiments at RIKEN.

\section{References}

[1] Lee T D and Yang C N 1952 Phys. Rev. 87410

[2]Lee Koo-Chul 1994 Phys. Rev. Lett. 732801

[3] Lee Koo-Chul 1996 Phys. Rev. E 536558

[4] Fisher M E 1967 Rep. Prog. Phys. 30615

[5] Yang C N and Lee T D 1952 Phys. Rev. 87404

[6] Kirkham J E and Wallace D J 1979 J. Phys. A: Math. Gen. 12 L47 
[7] Fisher M E 1978 Phys. Rev. Lett. 401610

[8] Lai S N and Fisher M E 1995 J. Chem. Phys. 1038144

[9] Binek Ch 1998 Phys. Rev. Lett. 815644

[10] Henkel M 1999 Conformal Invariance and Critical Phenomena (Berlin: Springer) p 23

[11] Dillon J F, Chen E Y and Guggenheim H J 1978 Phys. Rev. B 18377

[12] De Jongh L J and Miedema A R 1974 Adv. Phys. 231

[13] Birgeneau R, Yelon W B, Cohen E and Makovsky J 1972 Phys. Rev. B 52607

[14] Kortman P J and Griffiths R B 1971 Phys. Rev. Lett. 271439

[15] Wang Xian-Zhi and Kim Jai Sam 1998 Phys. Rev. E 575013

[16] Wang Xian-Zhi and Kim Jai Sam 1998 Phys. Rev E 584174

[17] Stanley H E 1971 Introduction to Phase Transitions and Critical Phenomena (New York: Oxford University Press)

[18] Himpsel F J, Ortega J E, Mankey G J and Willis R F 1998 Adv. Phys. 47511

[19] Kohlhepp J, Elmers H J, Cordes S and Gradmann U 1992 Phys. Rev. B 4512287

[20] Li Y and Baberschke K 1992 Phys. Rev. Lett. 681208

[21] Farle M 1998 Rep. Prog. Phys. 61755

[22] Hucht A and Usadel K D 1997 Phys. Rev. B 555579

[23] Baberschke K 1996 Appl. Phys. A 62417 